\title{
Restructuring of emergent grain boundaries at free surfaces - an interplay between core stabilization and elastic stress generation
}

\section{Xiaopu Zhang ( $\nabla$ xiaopuz@tcd.ie)}

School of Chemistry, Trinity College Dublin

\section{Mengyuan Wang}

University of Science and Technology of China

Hailong Wang

University of Science and Technology of China

Moneesh Upmanyu

Northeastern University

John Boland

School of Chemistry, Trinity College Dublin, Dublin 2

Article

Keywords:

Posted Date: January 7th, 2022

DOI: https://doi.org/10.21203/rs.3.rs-1235115/v1

License: (c) (1) This work is licensed under a Creative Commons Attribution 4.0 International License. Read Full License 


\title{
Restructuring of emergent grain boundaries at free surfaces - an interplay between core stabilization and elastic stress generation
}

\author{
Authors: Xiaopu Zhang, ${ }^{*}$ Mengyuan Wang, ${ }^{2}$ Hailong Wang, ${ }^{2 *}$ Moneesh Upmanyu, ${ }^{3 *}$
}

John J. Boland ${ }^{1 *}$

\begin{abstract}
Affiliations:
${ }^{1}$ Centre for Research on Adaptive Nanostructures and Nanodevices (CRANN), AMBER SFI

Research Centre and School of Chemistry, Trinity College Dublin, Dublin 2, Ireland

${ }^{2}$ CAS Key Laboratory of Mechanical Behavior and Design of Materials, Department of

Modern Mechanics, University of Science and Technology of China, Hefei, Anhui 230027, People's Republic of China

${ }^{3}$ Group for Simulation and Theory of Atomic-Scale Material Phenomena (stAMP), Mechanical and Industrial Engineering, Northeastern University, Boston, MA 02115, USA

*Correspondence to:
\end{abstract}

jboland@tcd.ie,xiaopuz@tcd.ie,

m.upmanyu@northeastern.edu \& hailwang@ustc.edu.cn

\begin{abstract}
Scanning tunneling microscopy and numerical calculations are used to study the structure and relaxation of grain boundaries at the surface of planar nanocrystalline copper (111) films and bicrystals. We show that the strong energetic preference for boundary cores to lie along closepacked planes introduces a restructuring that rotates adjoining grains and generates elastic stresses in the triple junction region. The interplay of this stress field and the core stabilization determines the length scale of the restructuring and controls the shape and magnitude of the displacement field around the triple junction. Depending on the in-plane angle, restructured boundaries can extend to depths of $\sim 15 \mathrm{~nm}$ with the associated elastic stress fields extending to even greater depths. These results point to a new mechanism of boundary relaxation at surfaces that is expected to play an important role in grain coalescence and stress evolution in growing films.
\end{abstract}

\section{Main}

\section{Introduction}

Grain boundaries (GBs) impact a wide range of properties - electrical transport in nanoscale materials ${ }^{1}$, intergranular corrosion and catalysis at surface ${ }^{2-5}$, stress evolution in thin films 6,7 , and the mechanical strength of polycrystalline materials ${ }^{8}-$ all critical to materials and device technologies ${ }^{9-12}$. The ever-increasing demand for innovation has heightened the need for an improved understanding of GBs and their impact on performance ${ }^{13-15}$. However, little is known about the atomic-scale behavior of emergent grain boundaries (eGBs) at the surfaces of macroscopic materials. These studies are particularly challenging due to surface corrosion phenomena and the potential of capping layers used in TEM to suppress the intrinsic behavior of eGBs. Thus, it is challenging for TEM to detect atomic relaxation along the axis of eGBs or the natural shape of the triple junction (TJ) so that the eGB behavior and their associated surface and size effects have largely gone unstudied experimentally ${ }^{16,17}$. Alternatively, the structure of eGBs is known to control dynamical thermal grooving at high temperatures ${ }^{18-20}$, but is poorly understood at lower temperatures where structure and stress play important roles. 
Here we studied the structure of eGBs on surfaces of copper (111) nanocrystalline films and bicrystals, using of UHV cryogenic STM together with numerical calculations. The picometer resolution of STM allows a precise mapping of the atomic structure of eGBs and the displacement field of the free surface at the TJ. We identify the existence of a restructuring phenomenon that is driven by an energetic preference for boundary cores to lie along closepacked planes (CPPs) that involves the rotation of adjoining grains and the generation of elastic stresses in the $\mathrm{TJ}$ region. The interplay of the core stabilization and this stress field determines the length scale of the restructured boundary and controls the shape and magnitude of the displacement field around the TJ.

\section{Atomic \& nanoscale structures and geometrical analysis}

We begin by considering the atomic structure of an eGB on a $50 \mathrm{~nm}$ thick nanocrystalline copper (111) film. Details of the sample, preparation and STM measurement are provided in the Methods. Figure 1a shows the perspective view of the TJ, where the boundary meets the surface. The grains on either side of the boundary are about $20 \mathrm{~nm}$ wide and TJ is located in a local groove. Fig. 1b shows an atomic resolution image of this boundary. Both sides are clearly (111) surfaces as expected and eGB cores show dislocation-like features similar to the emergent LAGB previously reported ${ }^{21}$. Although the atomic planes are curved or rotated downwards into the GB plane, there is no evidence of step formation, consistent with a free surface that is able to respond to the presence of a stress field in the subsurface region. The absence of surface steps confirms that the groove is not related to the Mullins' thermalgrooving phenomenon and Wulff construction ${ }^{18,19,22}$.

The misorientation or in-plane angle $\theta$ measured in Fig. $1 \mathrm{~b}$ between the [1 $\overline{1} 0]$ directions of the two adjoining grains is $\sim 25^{\circ}$. In the half period of the boundary, the Burgers circuit in green shows that there is a $\vec{B} / 2=[1 \overline{1} 0]$ closure failure along half of the period vector $\vec{p} / 2=$ $5 / 4[\overline{1} \overline{1} 2]+2 / 4[1 \overline{1} 0]$, one with a spacing $3 / 4[\overline{1} \overline{1} 2]+1 / 4[1 \overline{1} 0]$ and another with a spacing $2 / 4[\overline{1} \overline{1} 2]+1 / 4[1 \overline{1} 0]$. When inserted into Frank's formula ${ }^{23}$

$$
\sin (\theta / 2)=B / 2 p
$$

we find $\theta=26.01^{\circ}$, which agrees well with our measurement of $\sim 25^{\circ}$. Hence, we will refer to this boundary as eGB26. Using the surface basis of $1 / 4$ [ $\overline{1} \overline{1} 2]$ and $1 / 4$ [1시 $]$, the period vector can be rewritten as $[10,4]$ in surface notation, which decomposes into $[10,4]=[3,1]+[2,1]+[3,1]+[2,1]$ or $[3,2,3,2]$ for short.

The nature of the restructured boundary is evident from an analysis of the displacement field around the TJ. The groove width and minimum depth measured across the local maxima along the TJ are $\sim 3 \mathrm{~nm}$ and $\sim 65 \mathrm{pm}$, respectively (Fig 1c) and groove volume is $\sim 16$ atoms per period. Away from the boundary on either side of the groove the grains recover and return towards the planar orientation. The local angle within the groove is $\sim 0.15 \mathrm{rad}$ or $\sim 8.6^{\circ}$, meaning that the atomic planes on either side of the boundary are rotated $4.3^{\circ}$ away from the film normal. The global angle beyond the TJ groove is $\sim 0.7^{\circ}$. Previously we showed that adjoining grains at low angle boundaries in nanocrystalline copper (111) films exhibit an outof-plane rotation angle $\varphi$ that scales with the in-plane misorientation angle $\theta$ : $\tan (\varphi / 2)=1 / 2 \sqrt{2} \cdot \sin (\theta / 2)^{21}$. The driving force for out-of-plane rotation is the energy anisotropy of the dislocation line that favors a [112] tilt axis that stabilizes the GB cores by enabling them to lie long $\{111\}$ planes. For eGB26, the predicted rotation angle $\varphi / 2$ on each side of the boundary is $\sim 4.5^{\circ}$ and very close to the local $4.3^{\circ}$ groove angle measured in Fig. $1 \mathrm{c}, \mathrm{d}$. From the boundary geometry perspective ${ }^{21}$, a finite local groove angle implies that close to the surface the local tilt axis is shifted away from [111] towards [112], whereas the constant near-zero angle $\left(\sim 0.7^{\circ}\right)$ further away from the boundary implies that deep into the film the tilt 
axis returns to [111]. Different tilt axis have different symmetry, which indicates a phase-like restructuring around the TJ. We tested the generality of these results by mapping the TJ displacement field for a wide range of GB angles and found excellent agreement between the local groove angle and that predicted by the $(\theta / 2, \varphi / 2)$ scaling (see Fig. 1d). As expected, a finite positive (negative) global angle results in a smaller (larger) local angle compared to that predicted by geometry. Collectively, these results point to a strong preference for GB cores to lie along $\{111\}$ planes regardless of the GB misorientation angle (see below).

\section{Bicrystal \& surface effect}

To further confirm the generality of these results and that the shift of the tilt axis towards [112] is a property of eGBs in copper [111] materials, we employed STM to analyze the structure of the single boundary in an engineered macroscopic bicrystal. The bicrystal was 2 $\mathrm{mm}$ thick and $8 \mathrm{~mm}$ diameter and comprised of two $\mathrm{Cu}$ (111) single crystals that have been oriented and fused to form a GB $26.01^{\circ}$ boundary (MaTeck Material). The preparation of the bicrystal for STM analysis is described in the Methods.

Figure 2a shows the presence of the single boundary and the arrangement of surface steps on either side of this boundary. A close-up view image is shown in Fig. 2b, which was recorded at a location $>10 \mathrm{~nm}$ from the nearest step. Figure $2 \mathrm{~b}$ inset shows an atomic resolution image of the adjoining crystals of the bicrystal that confirms the (111) surface periodicity and that the in-plane angle is indeed 26.01 degrees. Importantly we note the period vector of the boundary decomposes into [3,2,3,2], identical to the decomposition observed in nanocrystalline film in Fig. 1b. The displacement field across the bicrystal boundary is shown in Fig. 2c. The TJ exists within a groove formed between the adjoining crystals. Topographic-sections across the groove recorded under different bias conditions (see Fig. SI-1) show evidence of an electronic effect at larger positive bias (also seen in nanocrystalline films), the width and depth of the groove is essentially identical to that recorded for the nanocrystalline film. The local angle is $5.1^{\circ}$ while far from the groove the global angle is $\sim-0.5^{\circ}$. The existence of near identical eGBs at the surfaces of $50 \mathrm{~nm}$ thick nanocrystalline films and macroscopic bicrystals demonstrates that the restructured eGB26 is not influenced by the limited grain size $(\sim 50 \mathrm{~nm})$ in the film and that the length scale of restructured boundary is much smaller than this grain size.

\section{Bulk GB energy calculation}

STM provides a direct measurement of TJ structure and the displacement normal to the surface. To understand the boundary restructuring and associated subsurface deformation fields, we rely on numerical calculations. Since for LAGBs the shift of the tilt axis towards [112] leads to a reduction of the boundary energy ${ }^{21}$, we calculated the corresponding driving force for a wide range of in-plane angles, i.e., the energy difference $\Delta \gamma$ between a bulk GB with a tilt axis [111] and its corresponding bulk boundary with the tilt axis shifted toward [112], shown schematically as black and red in Fig. SI-2, respectively (GB geometrical specifications in Methods). Classical molecular statics (MS) calculations results in Fig. 3a show that for all in-plane angles $\theta$, the [112] tilt boundary is always lower in energy. For each in-plane angle, the boundary energy decreases smoothly as the tilt axis shifts across the range of possible tilt axes between [111] and [112], revealing the absence of a thermodynamic barrier to grain rotation. For copper, the values of $\Delta \gamma$ range from $70 \mathrm{~mJ} \mathrm{~m}^{-2}$ to $300 \mathrm{~mJ} \mathrm{~m}^{-2}$ depending on the misorientation angle $\theta$ and in all instances $\Delta \gamma$ is a significant fraction of the original boundary energy. For GB $26.01^{\circ}, \Delta \gamma=112 \mathrm{~mJ} / \mathrm{m}^{2}$, or $\sim 14 \%$ of the GB $26.01^{\circ}$ energy of $833 \mathrm{~mJ} / \mathrm{m}^{2}$ (see Fig. SI-3).

\section{eGB calculation}


To see if the reduction in the bulk GB energy results in GB restructuring at the surface, we perform all-atom MS and molecular dynamics (MD) simulations of the symmetric tilt eGB26 in an $H \geq 50 \mathrm{~nm}$ thick [111] copper film (Methods and Tab. SI-1). While we observe a narrower and shallower groove compared to experiment (depth $<10 \mathrm{pm}$ and full width at half maximum $(\mathrm{FWHM})<1 \mathrm{~nm}$ ) at a $[3,2,3,2]$ decomposed surface TJ, the structural transition of the boundary is absent (Figure SI-4). The change in energy of the TJ in the eGB26 is negligible (Supplementary Note and Fig. SI-7). Alternatively, mechanical reorientation of the rotated eGB[112]26 onto a (111) substrate preserves the [112] misorientation axis and the TJ structure (see Fig. SI-6). While the eGB energy now increases due to the (elastic) stresses associated with the reorientation, the surface profiles show increasing agreement with the experimental profiles (Fig. 1c \& 2c) as the thickness of the reoriented eGB[112]26 is decreased to below $10 \mathrm{~nm}$ (Fig. SI-8). This is suggestive of partial rotation of the top layer and is consistent with a simple dimensional analysis: for a reoriented film of total height $H$, the elastic energy cost scales as volume $\left(\sim H^{2}\right)$ while the GB energy reduction is proportional to its area $(\sim H \Delta \gamma)$.

We study the stability of the partially rotated eGB system using a modified computational framework eGB system summarized in Fig. 3b (see Methods). The rotation is now limited to a top layer of varying thickness $h$ and consisting of a $\mathrm{V}$-shaped notch with an included angle $\varphi$. Commensurability between the rotated and reference (unrotated) regions of the film is ensured by shear-stitching the notch along the boundary normal, resulting in a valley at the eGB. Analogously, shear-packing of the cutout wedge at the eGBs leads to ridges at the GBs that bound the cell edges. The shear stitching/packing scheme is equivalent to the rotation of the top layer to eliminate the notch/wedge and mate it with the rest of the crystal, as summarized in Fig. SI-9.

\section{eGB subsurface structure}

Figure 4a shows the atomic-scale TJ structure following an equilibrium MS simulation of a $h=1.25 \mathrm{~nm}$ [6 (111) layers] thick rotated layer within an $H=50 \mathrm{~nm}$ thick film. The corrugation along the groove is evident from the surface profiles extracted at local maxima and minima, labelled X1 and X2 respectively. The corresponding line profiles plotted in Fig. 4b reveal groove depths of $D 1 \approx 100 \mathrm{pm}$ and $D 2 \approx 40 \mathrm{pm}$ respectively, and a surface width (FWHM $\sim 1.5 \mathrm{~nm}$ ) that is at least 50\% larger compared to the unrotated eGB and similar to experimental FWHM of $1.3 \mathrm{~nm}$. The local angle at the minima is $\sim \varphi$ and the global angle asymptotes to zero over $\mathrm{a} \sim 10 \mathrm{~nm}$ width centered at the eGB (Fig. $4 \mathrm{~b}$ ). The periodic TJ structure shows the emergence of dislocation-like features with a decomposition consistent with experiments (top, Fig. 4c). Subsurface characterization reveals co-existence of the two distinct GB structures with a transition at approximately the prescribed layer thickness $h$ (bottom, Fig. $4 \mathrm{c})$. This restructured eGB is stable to preparing temperatures ( $~ 800 \mathrm{~K})$. Varying $h$ at fixed film thickness $(H=50 \mathrm{~nm})$ leads to similar groove profiles, albeit with differing TJ widths (Fig. SI-10). The energy of each of these partially rotated eGBs is lower than that of the rotated and reoriented film, indicating that the equilibrium top layer thickness is less than 50 $\mathrm{nm}$, consistent with the size independent local groove angles observed in the experiments.

\section{eGB energetics: driving force for $e G B$ restructuring and its stability}

We analyze the stability of the partially rotated eGB system using a combination of scaling analyses and continuum computations. For a (111) copper film with surface energy $\gamma_{s}$, the driving force for rotation (per film width) to form a valley is (Supplementary Note)

$$
\Delta U_{F}=\left[\gamma_{[112]} / \cos (\varphi / 2)-\gamma_{[111]}\right] h-\left[2 \gamma_{S} \cdot \tan (\varphi / 2)\right] h
$$


where the first term is the contribution from the reduction in the GB area and the second term is the surface area eliminated by shear-stitching the notch 1 . The elastic deformation energy scales quadratically with the notch height $h$ and shear strain $\varphi$. Detailed finite element method (FEM) computations of a shear-stitched surface notch (see Methods) reveal a top layer under tension (Fig. SI-11) and a net elastic energy of the form

$$
\Delta U_{e}=f(h / H) C(v) G(\varphi h)^{2}
$$

where $G$ is the shear modulus, $C$ is a material constant set by the Poisson's ratio $v$, and the function $f$ is the correction due to the finite thickness of the film parametrized by the ratio $h / H$ (Fig. SI-12 and Supplementary Note).

Figure $4 d$ shows the layer thickness dependence of these energetic contributions for the eGB26 system with film thickness $H=50 \mathrm{~nm}$. The relevant interfacial and bulk parameters for pure copper are listed in Tab. SI-3 5. The elastic energy curve is plotted as a range based on anisotropy in the elastic parameters of pure copper $(G, v)$ along and normal to the film, including the average over all orientations in a randomly textured polycrystal. Minimization of the total energy $\Delta U_{F}+\Delta U_{e}$ yields an equilibrium top layer thickness $h^{*}=1.01 \mathrm{~nm}$, or approximately five (111) layers, and a net energy change $\Delta U^{*}=-0.1547 \mathrm{~nJ} / \mathrm{m}$. The minimum corresponds to elastic parameters along the GB normal and is close to that based on a polycrystalline average $\left(h^{*}=1.00 \mathrm{~nm}\right)$, see Tab. SI-5.

The continuum analysis is performed for a single crystal, and therefore ignores higher order corrections, if any, due to stress accommodation at the GB. Surface stresses and stressdependent GB energies can drive additional changes. We capture these corrections using allatom computations with rotated layer thickness in the range $0 \leq h \leq 2 \mathrm{~nm}$, or $0-$ $9\{111\}$ layers. The variation in the total energy of the eGB system, also plotted in Fig. $4 \mathrm{~b}$ for comparison, is similar to the continuum predictions with a slight shift in the minimum to $h^{*}=$ $1.25 \mathrm{~nm}$. The energy change $\Delta U^{*}=-0.0954 \mathrm{~nJ} / \mathrm{m}$ is smaller, likely due to the discrete nature of the $\{111\}$ layers and stress-dependent bulk and interfacial parameters that are naturally incorporated in the atomistic model. This interplay is evident in the atomic stress distribution in Fig. 4e that exhibits a tensile to compressive stresses at the line defect that separates the two co-existing GBs, consistent with the elastic healing of a V-shaped notch (Fig. SI-11), and is distinct from the intrinsic stresses associated with each GB.

\section{eGB generalization to LAGB (energetics and subsurface structure)}

We test the generality of the energetic interplay at eGBs by performing a similar analysis for a valley formed at the eGB3.89 system (Fig. 5). A prescribed out-of-plane rotation of $\varphi=$ $1.375^{\circ}$ corresponds to the [111] $\rightarrow[112]$ shift of the misorientation axis (Tab. SI-1). The GB and surface contributions to the driving force together with FEM-based bulk elastic energy are plotted in Fig. 5a. The surface contribution for the LAGB is smaller, as expected. The net energy exhibits a minimum at $h^{*} \cong 13 \mathrm{~nm}$ (Tab. SI-5) with negligible size effects at larger film thicknesses in the range $H=50-100 \mathrm{~nm}$. MS computations of the eGB3.89 system around this minimum mimic the trends observed in the continuum computations, with an equilibrium rotated layer thickness that is shifted to $h^{*}=14.4 \mathrm{~nm}$. The energy change of eGB3.89 system $\Delta U^{*}=-0.51 \mathrm{~nJ} / \mathrm{m}$ is again smaller than the continuum computations $\Delta U^{*}=-0.67 \mathrm{~nJ} / \mathrm{m}$.

The surface profile shows corrugation with $D_{1} \cong 110 \mathrm{pm}$ and $D_{2} \sim 40 \mathrm{pm}$, similar to the $\mathrm{STM}$ profiles in NC films with small deviations $\sim 7.5 \mathrm{~nm}$ away from TJ (Fig. 5b). The

\footnotetext{
${ }^{1}$ Both contributions are dependent on the out-of-plane rotation $\varphi$, and therefore the misorientation angle $\theta$ $(\tan \varphi / 2 \propto \sin \theta / 2)$. For LAGBs, the driving force approaches $\Delta \gamma$ and the surface contribution becomes increasingly important for HAGBs.
} 
corrugation $\sim 70 \mathrm{pm}$ is indicative of dissociated partial dislocations. The surface TJ exhibits a $1 / 2$ [ $\overline{9} \overline{8} 17]$ period vector and the SF ribbon planes lie on the CPP, consistent with [112] tilt axis. The elastic stresses due to the reorientation increase the width of the SF ribbons at the surface (Fig. SI-13), suggestive of a GB contribution to the stress accommodation. Both experimental and atomistic peak $\left(X_{2}\right)$ profiles reveal additional reconstructions at the SF edges at the surface that subvert the complete formation of the local groove. Figure $5 \mathrm{c}-\mathrm{d}$ show the subsurface structure of the LAGB and stress distribution around the eGB. We see a sharp transition between the two co-existing GB structure, stabilized by a diffuse (compressive) stress, and the transition from tensile to compression stresses occurs well away from the line defect separating the two GB structures. The overall interplay between geometry and mechanics is similar to the observations for eGB26. The excellent agreement between atomistics and continuum computations for both eGBs indicates that higher order effects have a minimal effect on the energetics that sets the thickness of the rotated layer.

\section{Concluding remarks}

In this paper we demonstrated the existence of a natural driving force that restructures GBs at surfaces. This phenomenon will be particularly important whenever there is an interplay between GB energy, structure and stress. For example, tensile or compressive stresses generated in films at different growth stage ${ }^{24,25}$ will modulate the intrinsic stress of eGBs, decreasing (increasing) the length scale at valley-shaped (ridge-shaped) boundaries, respectively. It will also impact material properties controlled by GBs, particularly in ultrathin films with thicknesses approaching the length scale of the restructured boundary and stress fields. In this instance, the film is comprised of a network of restructured boundaries and the surface effect described here becomes a size effect. Since [112] lies within a CPP, any boundary with this tilt axis can be considered to be a folded CPP. Given that electronic transport in copper occurs predominantly along CPPs ${ }^{26,27}$ these [112] boundaries should exhibit reduced levels of boundary scattering. To realise these benefits it is necessary to develop processes that enable the deposition of materials with thermodynamically-relaxed [112] tilt boundaries. 
Figure 1 Grain boundary structures at atomic resolution and nanoscale.

a. Perspective view of GB $26.01^{\circ}$. b. atomic resolution mapping of GB $26.01^{\circ}$. The red lines show the vectors in both half crystals. The green path shows a Burgers circuit with a closure failure of a content two times nearest-neighbor distance. c. STM topography of GB $26.01^{\circ}$. d. Data points of white and green lines in (c). The black line is a local fit of the green points and the red line is the first derivative of the black line. Tunneling parameters for (c) are $\mathrm{I}=20 \mathrm{pA}$ and $U=-500 \mathrm{mV}$. Tunneling parameters for (b) are $\mathrm{I}=30 \mathrm{pA}$ and $\mathrm{U}=5 \mathrm{mV}$. (d) The local and global out-of-plane angles as a function of in-plane angles for LAGBs and HAGBs. Note that GB [111] $18.74^{\circ}$ shows a range of the out-of-plane displacement angles at different sites.
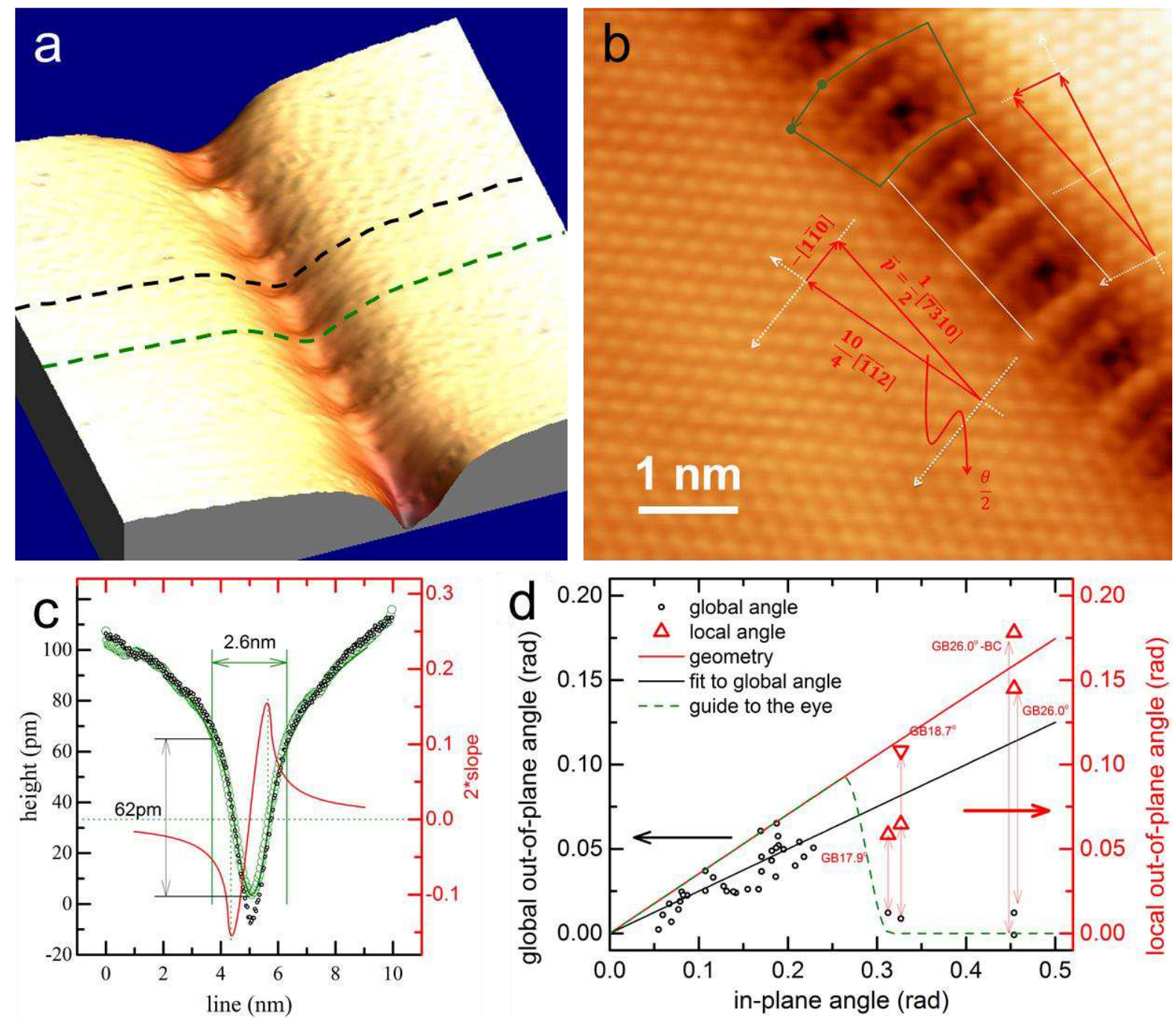
Figure 2 Grain boundary structure at surface in bicrystal.

a. STM topography image shows a straight line, where GB $26.01^{\circ}$ emerges at the bicrystal (BC) surface. b. STM image of eGB26 at the surface. Inset, shows atomic resolution of the TJ region and confirms the $[3,2,3,2]$ decomposition. c. Topographic profile of the TJ groove recorded along the cross section in (b) together with the profile of the TJ groove at the nanocrystalline (NC) surface. Tunneling parameters for (a) are $\mathrm{I}=20 \mathrm{pA}$ and $\mathrm{U}=2 \mathrm{~V}$, for (b) inset are $\mathrm{I}=20$ $\mathrm{pA}$ and $\mathrm{U}=20 \mathrm{mV}$, for (b) are $\mathrm{I}=20 \mathrm{pA}$ and $\mathrm{U}=-200 \mathrm{mV}$.
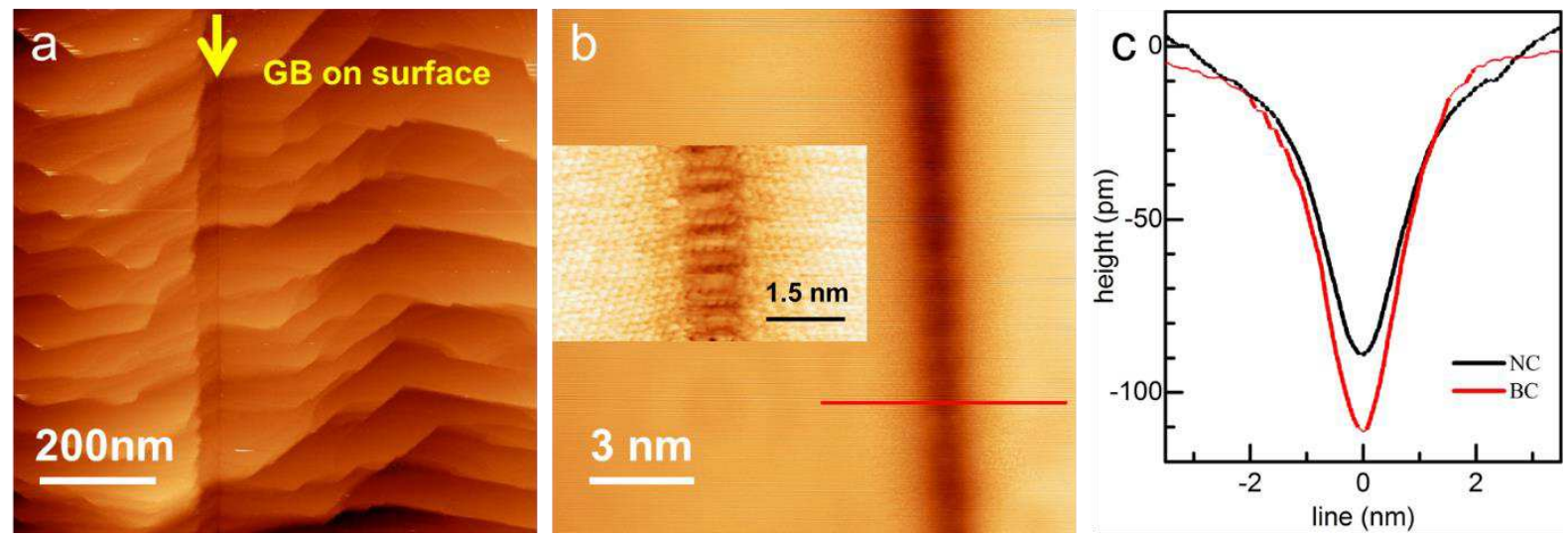

\section{Figure 3 GB energy and eGB calculation.}

a. In-plane angle dependent GB energy as the composite axis shift from [111] to [112] through out-of-plane rotation. b. Computational scheme used to study the partial rotation of a top layer of thickness $\boldsymbol{h}$ (shaded green) within a film of thickness $\boldsymbol{H} \gg \boldsymbol{h}$. Solid lines indicate GBs separated by the grain size $\boldsymbol{L}$. Red and blue lines correspond to the unrotated and rotated GBs, respectively. The location of the valleys and ridges are as indicated.
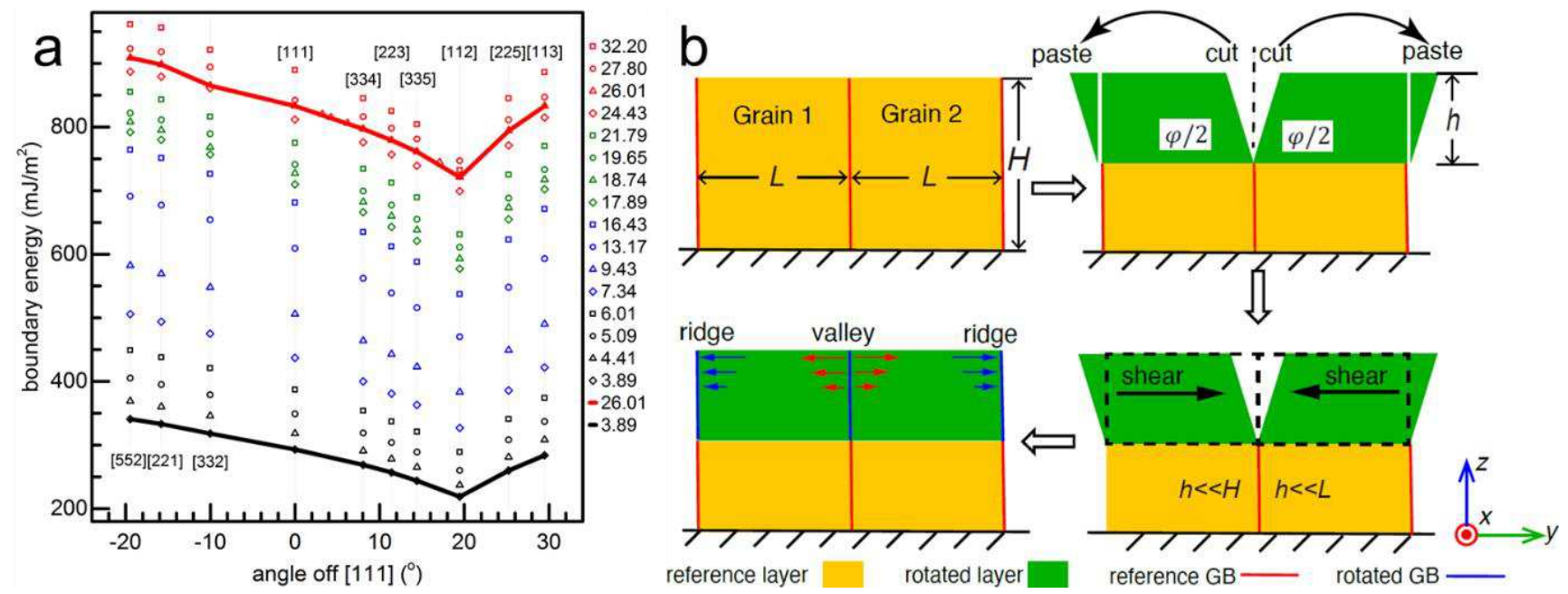
(a) Atomic-scale configuration of an equilibrated eGB composed of the $\boldsymbol{\theta}=\mathbf{2 6 . 0 1}{ }^{\circ}$ HAGB with a rotated layer thickness of $1.25 \mathrm{~nm}$, or $6\{\mathbf{1 1 1}\}$ layers in a $\boldsymbol{H}=\mathbf{5 0}$ thick (111) copper film. Atoms are coloured based on their depth coordinate. (b) Line profiles along troughs (blue, $\boldsymbol{X}_{1}$ ) and peaks (red, $\boldsymbol{X}_{2}$ ) within the undulating surface TJ, as indicated in (a). The solid green line is the slope of the $\boldsymbol{X}_{\mathbf{1}}$ profile. (c, top) Magnified view of the surface TJ. The dashed green lines connecting close-packed directions across the eGB serve as guides for the planar decomposition of the indicated periodicity vector. Atoms are coloured based on the potential energy (c, bottom). Side view showing the through thickness GB structure within the rotated and reference layer. The defect atoms are identified and coloured based on the central symmetry parameter. (d) The GB, surface and elastic energy contributions to the total energy of the eGB for varying rotated layer thicknesses. The elastic energy cost is plotted as a range based on continuum (FEM) computations; see text for details. Green spheres are results of MS simulations. The minima corresponding to the continuum and atomistic plots are indicated. (e) Through-thickness stress distribution in the vicinity of the eGB. The color indicates the atomic (virial) stress.
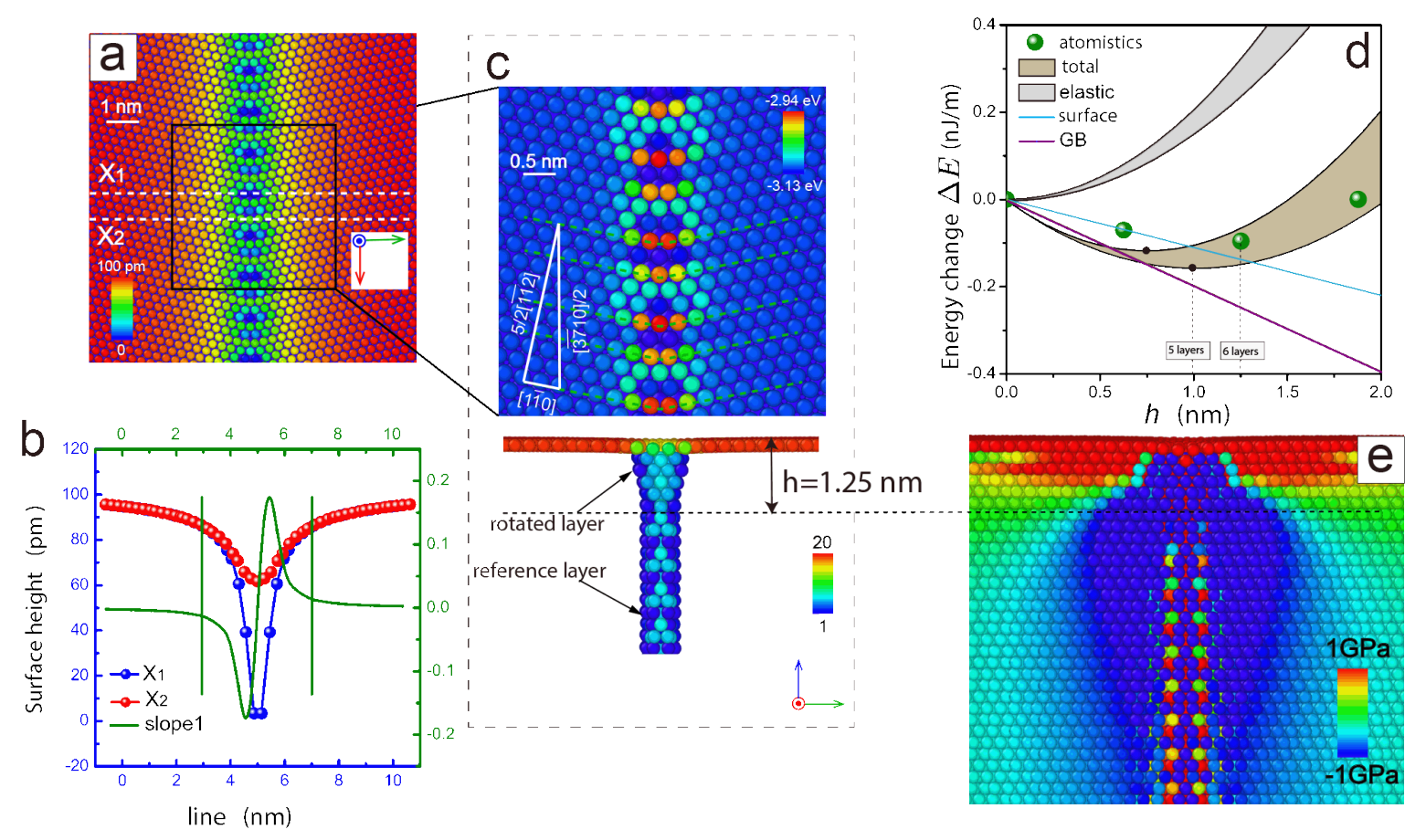
Figure 5 Atomistic-continuum computational analyses for the eGB3.9 system.

(a) Same as in Fig. 4d, but for eGB composed of the $\boldsymbol{\theta}=\mathbf{3 . 8 9 ^ { \circ }}$ LAGB. The vertical dashed lines indicate the minima for the continuum and atomistic computations. (b) The trough (green) and peak (red) line profiles extracted via MS simulations (spheres) and STM characterization of a $50 \mathrm{~nm}$ thick NC copper film (solid lines). (c-d) Through thickness (c) GB structure using the central symmetry parameter and (d) stress distribution based on the atomic stress observed.

a

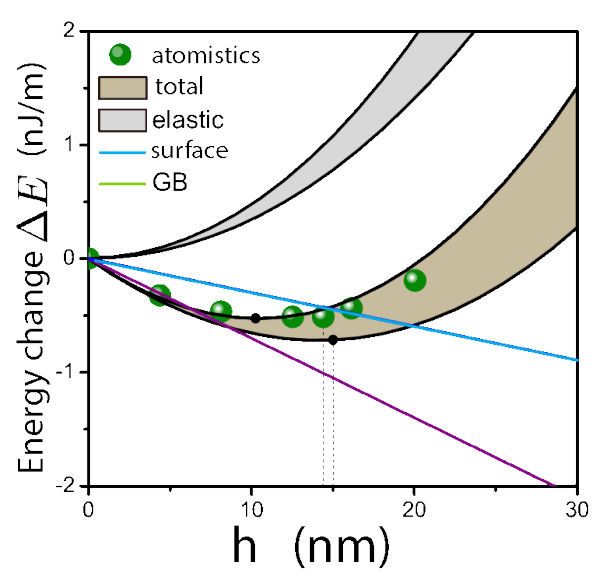

C

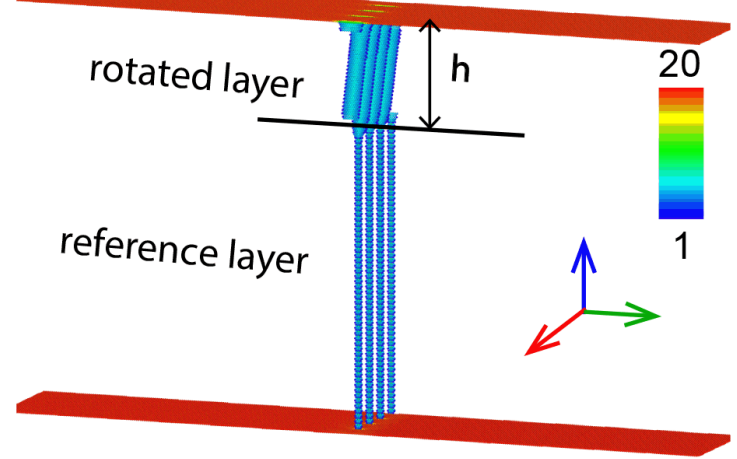

b

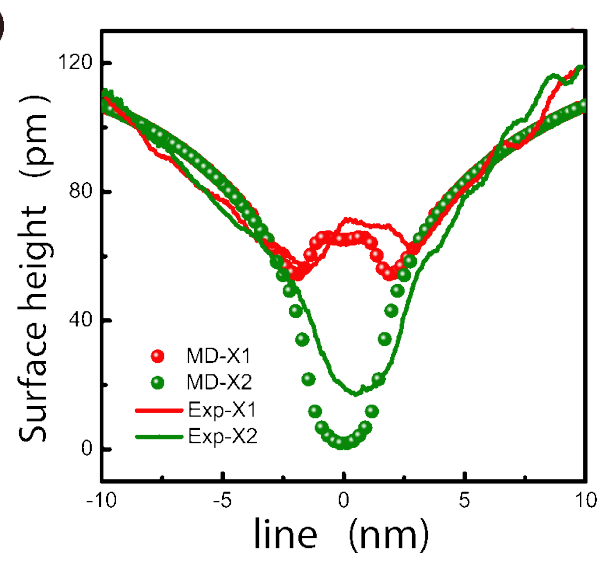

d

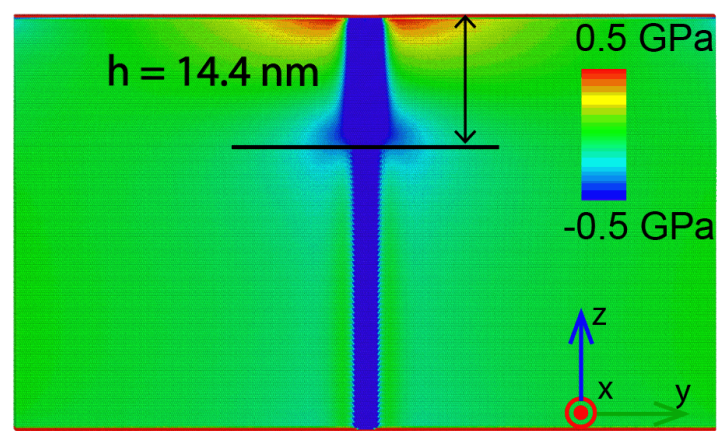


310 J.J.B. conceived and initiated the study. J.J.B. and X.Z. designed the experiments on 311 nanocrystalline and bicrystal systems. X.Z. carried out all experiments, calculated all 312 boundaries in the bulk. J.J.B. and X.Z. analyzed the triple junction geometry and the change in 313 the composite rotation axis across the boundary.

314 M.U and H.W. designed the atomic-scale \& continuum computations and developed the 315 computational scheme for generation and analyses of rotated bicrystals and grain boundary 316 structures. H.W. and M.W. performed the atomic-scale simulations for equilibrium defect 317 structure and energetics, and surface configurations. H.W. and M.W. performed the continuum 318 computations and scaling analyses for the rotated layer thicknesses.

319 X.Z., H.W, M.U, J.J.B discussed the results from experiments and calculations and wrote the 320 manuscript.

\section{Acknowledgments}

322 J.J.B and X.Z. acknowledge support from Science Foundation Ireland grants (12/RC/2278 and 323 16/IA/4462) and early discussions with David Srolovitz, Adrian Sutton and Jian Han, and thank 324 Peter Gleeson and John J. Plombon from Intel Corporation for the PVD samples.

325 H.W. and M.W. acknowledge support from the National Natural Science Foundation of China 326 (Grant No. 12172347) and the Fundamental Research Funds for the Central Universities (Grant 327 No. WK2480000006).

\section{Additional information}

329 Requests for materials should be addressed to X.Z. and H.W. 


\section{Methods}

\section{Sample preparation and scanning tunneling microscopy}

Nanocrystalline copper films are prepared from a 12 inch Ta coated ( $7 \mathrm{~nm}$ thick) Si wafer at room temperature. The sample preparation method can be found in our previous paper ${ }^{21}$. Engineered copper bicrystal $\mathrm{Cu}-\mathrm{BC}$ sample was prepared by MaTeck $\mathrm{GmbH}$ according to our design. It has a tilt axis [111], $\sim 26^{\circ}$ misorientation, mean-boundary-plane [ $\left.1 \overline{1} \overline{1} 2\right]$, and polished along the (111) surface. The sample was cut into a cylinder of $8 \mathrm{~cm}$ diameter and $2 \mathrm{~mm}$ thick, which are further cut into hat-shaped samples for sample installation. The bicrystal sample was cleaned by repeated cycles of Ar+ ion sputtering and annealing to $\sim 800 \mathrm{~K}$, respectively ${ }^{28}$. The clean surface with low impurity concentration is checked by STM.

\section{Calculation of boundaries in bulk}

We first define the median lattice as shown in Fig. SI-2a. Then the in-plane rotation, shown in Fig. SI-2a\&b, is introduced. Lastly, we add the out-of-plane rotation as shown in Fig. SI-2b. We calculated the boundaries in bulk using molecular statics with periodic boundary conditions in three direction, and built each repeat cell with a pair of parallel GBs of equal and opposite misorientation ${ }^{29}$. Structure searching were done with hundreds of boundary structures and only the lowest energy are reported in this paper ${ }^{23,30}$. Detailed description of geometrical specification and calculation of boundaries in bulk can be found in a separate paper $^{21}$.

Similarly, we also built symmetric tilt boundaries with tilt axis [111], mean boundary plane (1112) for different in-plane angles. The calculated boundary energy as a function of in-plane rotation angle are shown in red in Fig. SI-3a. Therefore, we only considered the boundaries with (11̄0) mean boundary plane, to analyze the experimental data.

\section{Atomic-scale simulations of emergent grain boundaries}

Bicrystallography: The study is limited to [111] symmetric tilt GBs in (111) orientated $\mathrm{Cu}$ thin films. Figure SI-5a b shows the in- and out-of-plane orientations of the crystals abutting the emergent grain boundary in a (111) orientated $\mathrm{Cu}$ thin film. The rotation of the misorientation axis is illustrated in Fig. SI-5c. It follows a pure geometric relation with the inand out-of-plane orientations ${ }^{21}, \tan (\varphi / 2)=\sin (\theta / 2) \tan (\psi)$. The crystal parameters for the $\theta=26.008^{\circ} \mathrm{HAGB}$ and the $\theta=3.890^{\circ}$ LAGB that form the basis for this study are tabulated in Tab. SI-1.

Bulk grain boundary structure: The molecular statics simulations are performed using LAMMPS $^{31}$ and a well-known embedded atom-method potential fit to a range of thermodynamic, kinetic and mechanical properties of $\mathrm{Cu}^{32}$. Periodic boundary conditions (PBCs) are enforced within the GB plane ( $x$ and $z$ directions, see Fig. 3b). For reference and rotated GBs, the sizes of the computational cells are reported in Tab. SI-1. Note that to the capture the periodicity of the discrete dislocations within the LAGB, larger computational cells are employed. For both GBs, size effect studies with larger and smaller cross-sectional areas were performed to ensure that the i) cohesive energy per atom of the cell $u_{c}$ and ii) the excess grain boundary energies do not exhibit any artifacts due to size effects.

For each GB with prescribed misorientation as well as orientations of the misorientation axis and grain boundary plane, several trial configurations are generated. The energy minimization of the structure is limited to atoms within a $10 \mathrm{~nm}$ wide region centered at the boundary plane, designated as the grain boundary atoms. Each trial configuration is first locally minimized using trial moves $(\sim 0.01 \mathrm{~nm}$ in a random direction) and the minimum structures are selected using a genetic algorithm. These relaxed configurations serve as the basis for a global minimization algorithm based on an energy criterion, wherein are atoms are deleted (or 
added) based on their potential energy with their nearest neighbors. The algorithm represents a zero-temperature extension of the grand canonical Monte Carlo algorithm for extracting grain boundary structures at finite temperatures. The local and global minimization is done iteratively in series until the grain boundary structure converges.

Emergent grain boundary structure and energetics: The PBCs are now limited to along the triple junction (x-direction) such that the top surface remains free. The normal displacements of remaining sides of the cell are fixed. Following the computational scheme shown in Fig. 3b, the V-shape notch layer with rotated GB (green) and the flat reference layer (yellow) are mated together such that there is no new GB formation between these rotated and reference layers. The entire structure is relaxed at highest preparing temperatures $(\sim 800 \mathrm{~K})$ using molecular dynamics (Nose-Hoover thermostat, 1 fs time step), and then equilibrated to zero temperature to extract the equilibrium height of the rotated layer.

Surface and cohesive energy: The surface energy is extracted by cleaving a perfect crystal along the (111) plane within molecular statics simulations and monitoring the change in energy of the computational cell. The excess energy normalized by the surface area is the surface energy (enthalpy), $\gamma_{s}$, and is consistent with past studies ${ }^{32}$. The cohesive energy $u_{c}$ is the energy per atom of a fully periodic computational cell of $\mathrm{FCC} \mathrm{Cu}$ atoms. The values are reported in Tab.SI-3.

Structural analyses: The atomic potential energy distributions, atomic stress virials and 3D central symmetry parameter are used to extract the structure of the bulk and emergent grain boundaries as well as the structures of the surface triple junctions. All calculations are performed within OVITO ${ }^{33}$.

Surface profiles: The surface profiles are extracted by tracking the z-coordinate of the capping (111) plane. In several instances, the profiles of the subsurface (111) are also monitored to extract the depth dependence of the surface groove.

\section{Continuum simulations}

Finite element simulations: The elastic field near the valley is extracted using the linear elastic constitutive law in the finite element method (FEM) package (ABAQUS 2017). The bicrystal film is modelled using 4-node bilinear plane strain quadrilateral elements (CPS4R in ABAQUS notation). Grains of identical dimensions are used to create the bicrystals (Fig. SI11). As in the atomic-scale simulations of the emergent grain boundaries, the normal displacements of sides and bottom of the computational cell are fixed while the surface remains free. To avoid the influence of boundary conditions, we set the bottom and sides far from the triple junction, $L / h=50, H / h=25$. Size Larger model sizes with $L / h=100, H / h=50$ result in a difference of the elastic energies that are less than $0.1 \%$ for both eGB systems.

Cut-paste-shear scheme: The V-shape notch of thickness $h$ along the grain boundary plane is created using a cut with an included angle $\varphi$. The elastic field is extracted by applying symmetrical stresses along the horizontal until the notch heals. Table SI-3 4 list the various combinations of elastic and interfacial parameters used in the continuum simulations ${ }^{34}$. The surface profiles and the displacement fields around the resultant valley are directly extracted by following the nodal displacements. 
1 Mayadas, A. F., Shatzkes, M. \& Janak, J. F. Electrical Resistivity Model for Polycrystalline Films - Case of Specular Reflection at External Surfaces. Appl Phys Lett 14, 345-\&, doi:Doi 10.1063/1.1652680 (1969).

2 An, D. et al. Correlating the five parameter grain boundary character distribution and the intergranular corrosion behaviour of a stainless steel using 3D orientation microscopy based on mechanical polishing serial sectioning. Acta Mater 156, $297-$ 309, doi:10.1016/j.actamat.2018.06.044 (2018).

3 Li, C. W., Ciston, J. \& Kanan, M. W. Electroreduction of carbon monoxide to liquid fuel on oxide-derived nanocrystalline copper. Nature 508, 504-507, doi:10.1038/nature13249 (2014).

4 Mariano, R. G. et al. Microstructural origin of locally enhanced CO2 electroreduction activity on gold. Nat Mater 20, 1000-+, doi:10.1038/s41563-021-00958-9 (2021).

5 Bettayeb, M. et al. Nanoscale Intergranular Corrosion and Relation with Grain Boundary Character as Studied In Situ on Copper. J Electrochem Soc 165, C835C841, doi:10.1149/2.1341811jes (2018).

6 Leib, J., Monig, R. \& Thompson, C. V. Direct evidence for effects of grain structure on reversible compressive deposition stresses in polycrystalline gold films. Phys Rev Lett 102, 256101, doi:10.1103/PhysRevLett.102.256101 (2009).

7 Vasco, E. \& Polop, C. Intrinsic Compressive Stress in Polycrystalline Films is Localized at Edges of the Grain Boundaries. Phys Rev Lett 119, 256102, doi:ARTN 256102

10.1103/PhysRevLett.119.256102 (2017).

8 Greer, J. R. \& De Hosson, J. T. M. Plasticity in small-sized metallic systems: Intrinsic versus extrinsic size effect. Prog Mater Sci 56, 654-724, doi:10.1016/j.pmatsci.2011.01.005 (2011).

9 Valencia, D. et al. Grain-Boundary Resistance in Copper Interconnects: From an Atomistic Model to a Neural Network. Phys Rev Appl 9, doi:ARTN 044005

10.1103/PhysRevApplied.9.044005 (2018).

10 Goodenough, J. B., Abruna, H. D. \& Buchanan, M. ANYL 28-Summary overview of basic research needs for electrical energy storage. Abstr Pap Am Chem S 234 (2007).

11 Wang, C. et al. Size-Dependent Grain-Boundary Structure with Improved Conductive and Mechanical Stabilities in Sub-10-nm Gold Crystals. Phys Rev Lett 120, 186102, doi:10.1103/PhysRevLett.120.186102 (2018).

12 Zhang, Y.-Y., Xie, H., Liu, L.-Z. \& Jin, H.-J. Surface Triple Junctions Govern the Strength of a Nanoscale Solid. Phys Rev Lett 126, 235501, doi:10.1103/PhysRevLett.126.235501 (2021).

13 Schiotz, J. \& Jacobsen, K. W. Nanocrystalline metals: Roughness in flatland. Nat Mater 16, 1059-1060, doi:10.1038/nmat5015 (2017).

14 Watanabe, T. Grain boundary engineering: historical perspective and future prospects. J Mater Sci 46, 4095-4115, doi:10.1007/s10853-011-5393-z (2011).

$15 \mathrm{Li}, \mathrm{X}$. \& Lu, K. Improving sustainability with simpler alloys. Science 364, 733-734, doi:10.1126/science.aaw9905 (2019).

16 Christiansen, J. et al. Atomic-scale structure of dislocations revealed by scanning tunneling microscopy and molecular dynamics. Phys Rev Lett 88, 206106, doi:10.1103/PhysRevLett.88.206106 (2002).

17 Frolov, T., Olmsted, D. L., Asta, M. \& Mishin, Y. Structural phase transformations in metallic grain boundaries. Nat Commun 4, 1899, doi:10.1038/ncomms2919 (2013). 
18 Mullins, W. W. Theory of Thermal Grooving. J Appl Phys 28, 333-339, doi:Doi

19 Srolovitz, D. J. \& Safran, S. A. Capillary Instabilities in Thin-Films .1. Energetics. $J$ Appl Phys 60, 247-254, doi:Doi 10.1063/1.337689 (1986).

20 Srolovitz, D. J. \& Safran, S. A. Capillary Instabilities in Thin-Films .2. Kinetics. J Appl Phys 60, 255-260, doi:Doi 10.1063/1.337691 (1986).

21 Zhang, X. et al. Nanocrystalline copper films are never flat. Science 357, 397-400, doi:10.1126/science.aan4797 (2017).

22 Herring, C. Some Theorems on the Free Energies of Crystal Surfaces. Phys Rev 82, 87-93, doi:DOI 10.1103/PhysRev.82.87 (1951).

23 Sutton, A. P. \& Balluffi, R. W. Interfaces in Crystalline Materials. (OUP Oxford, 2006).

24 Nix, W. D. \& Clemens, B. M. Crystallite coalescence: A mechanism for intrinsic tensile stresses in thin films. J Mater Res 14, 3467-3473, doi:Doi 10.1557/Jmr.1999.0468 (1999).

25 Pao, C. W., Foiles, S. M., Webb, E. B., 3rd, Srolovitz, D. J. \& Floro, J. A. Thin film compressive stresses due to adatom insertion into grain boundaries. Phys Rev Lett 99, 036102, doi:10.1103/PhysRevLett.99.036102 (2007).

26 Jones, S. L. T. et al. Electron transport properties of sub-3-nm diameter copper nanowires. Phys Rev B 92, doi:ARTN 115413

10.1103/PhysRevB.92.115413 (2015).

27 Weismann, A. et al. Seeing the Fermi surface in real space by nanoscale electron focusing. Science 323, 1190-1193, doi:10.1126/science.1168738 (2009).

28 Zhang, X. P. et al. Atomic superlattice formation mechanism revealed by scanning tunneling microscopy and kinetic Monte Carlo simulations. Phys Rev B 81, doi:ARTN 125438

10.1103/PhysRevB.81.125438 (2010).

29 Tschopp, M. A. \& McDowell, D. L. Asymmetric tilt grain boundary structure and energy in copper and aluminium. Philos Mag 87, 3871-3892, doi:10.1080/14786430701455321 (2007).

30 Han, J., Vitek, V. \& Srolovitz, D. J. Grain-boundary metastability and its statistical properties. Acta Mater 104, 259-273, doi:10.1016/j.actamat.2015.11.035 (2016).

31 Plimpton, S. Fast Parallel Algorithms for Short-Range Molecular-Dynamics. $J$ Comput Phys 117, 1-19, doi:DOI 10.1006/jcph.1995.1039 (1995).

32 Mishin, Y., Mehl, M. J., Papaconstantopoulos, D. A., Voter, A. F. \& Kress, J. D. Structural stability and lattice defects in copper: Ab initio, tight-binding, and embedded-atom calculations. Phys Rev B 63, 224106, doi:ARTN 224106 DOI 10.1103/PhysRevB.63.224106 (2001).

33 Stukowski, A. Visualization and analysis of atomistic simulation data with OVITOthe Open Visualization Tool. Model Simul Mater Sc 18, doi:Artn 015012 10.1088/0965-0393/18/1/015012 (2010).

34 Rassoulinejad-Mousavi, S. M., Mao, Y. J. \& Zhang, Y. W. Evaluation of copper, aluminum, and nickel interatomic potentials on predicting the elastic properties. $J$ Appl Phys 119, doi:Artn 244304 


\section{Supplementary Files}

This is a list of supplementary files associated with this preprint. Click to download.

- SupplementaryInformation.pdf 